\title{
Phase Difference Measurement Based on Recursive DFT with Spectrum Leakage Considered
}

\author{
Huiyue Yang ${ }^{1}$, Yaqing $\mathrm{Tu}^{2}$, Haitao $\mathrm{Zhang}^{3}$ and Ming $\mathrm{Li}^{4}$ \\ Department of Information Engineering, Logistical Engineering University, \\ Chongqing, China \\ huiyue_yang@163.com
}

\begin{abstract}
This paper investigates spectrum leakage influence on the performance of discrete Fourier transform (DFT) based phase difference measurement and proposes a novel method with spectrum leakage considered. In the proposed method, spectrum is firstly corrected by interpolation algorithm to remove the influence of short range leakage. To avoid negative frequency interference caused by the long range leakage, real-time phase difference is calculated in succession by a recursive DFT based algorithm with negative frequency contribution. And then, precision of the proposed method is theoretically demonstrated in detail. Furthermore, the method is applied to Coriolis mass flowmeter $(C M F)$. Simulation and experimental results show that the accuracy of phase difference measurement has largely improved, as compared with the existing method based on the sliding Goertzel algorithm.
\end{abstract}

Keywords: phase difference; spectrum leakage; discrete Fourier transform; negative frequency

\section{Introduction}

Phase difference measurement of cosine signals with the same frequency is an important topic in many measurement and signal processing tasks, such as radar, sonar, communication, electrical technology, power systems and industrial automation. In domain of high-precision flow measurement, Coriolis mass flow meter (CMF) operates properly by measuring the time interval of two vibration signals which is depended on the phase difference and frequency [1] A lot of methods for phase difference measurement have been developed to fulfill the comprehensive applications requirements.

In the existing methods, zero crossing detection based method [2] just requires small amounts of calculation, but poor in anti-interference ability and demands excessively for hardware. Digital correlation method [3] is good at suppressing random noise, but has difficult to eliminate harmonic interference. What's more, this method need to know the signal frequency in advance or required to maintain full period sampling which is often hard to realize in practice. Methods based on DFT [4] calculate phase difference by the subtraction of two DFT phases at the maximum spectral line. This method has good resistance to harmonic interference. However, an unfeasible technique of integral period sampling is also particularly needed [5-6]. Otherwise spectrum leakage takes place inevitability which has a passive effect for precision of phase difference measurement [7].

The Hilbert transform based method [8-9] which calculates phase difference by trigonometry operation between the signal and its Hilbert transform is proposed to detect variable phase difference. This method can figure out the phase difference at every sampling point in real-time and performs perfectly when the phase difference changes rapidly. However, it has feeble immune from interference, which limits practicalities of 
the method. A sliding Goertzel algorithm (SGA) [10] is also introduced to measure phase difference. But there is a slow convergence rate and a numerical overflow.

A sliding DFT is developed to measure the variable phase difference. To carry out the change of phase difference, a small number of sampled data are allowed to be taken in the DFT calculation. Then, spectrum leakage becomes inevitable and remarkable. However, the current phase difference measurement methods based on DFT spectrum analysis rarely consider the impact of spectrum leakage, especially the impact of negative frequency components [11]. This is why the results obtained either by the sliding DFT or by the sliding Goertzel algorithm deviate from the true values.

To solve the aforementioned problems, a novel recursive DFT method with spectrum leakage considered is proposed in this paper, which is expected to improve the accuracy of variable phase difference measurement and shorten the convergence stage of calculation.

This paper is organized into seven sections. In Section 2, principle of DFT-based phase difference measurement is deduced. In Section 3, how spectrum leakage reduces the precision of phase difference estimation is demonstrated from two aspects of short-range spectral leakage and long-range spectral leakage. Based on the recursive DFT, a novel method with spectrum leakage considered is proposed in Section 4. Precision of the proposed method is analyzed in Section 5. In Sections 6 and 7, results from simulations and experiments in $\mathrm{CMF}$ are given to validate the proposed method.

\section{Phase Difference Detection based on DFT}

The phase difference equals the subtraction of two DFT phases at the signal frequency. For two sine signals with the same frequency, the sampling sequences can be expressed as follows:

$$
\begin{aligned}
& s_{1 w}(n)=A_{1} \cos \left[2 \pi f_{0} n / f_{s}+\theta_{1}\right] \cdot w(n) \\
& s_{2 w}(n)=A_{2} \cos \left[2 \pi f_{0} n / f_{s}+\theta_{2}\right] \cdot w(n)
\end{aligned}
$$

Where $n=0,1, \cdots, N-1, A_{1}$ and $A_{2}$ are amplitudes, $\theta_{1}$ and $\theta_{2}$ are initial phases, $f_{s}\left(f_{s}>2 f_{0}\right)$ is sampling frequency and $f_{0}$ is the signal frequency . $w(n)$ is the discrete time window, whose length is $N$.

The DFT of $s_{1 w}(n)$ at $f_{0}$ can be expressed as:

$S_{1 w}(k)=\left[\frac{A_{1}}{2} e^{j \theta_{1}} \delta\left(f-f_{0}\right)+\frac{A_{1}}{2} e^{-j \theta_{1}} \delta\left(f+f_{0}\right)\right] W(f) e^{-j \frac{N}{2} f}=\frac{A_{1}}{2} W\left(f-f_{0}\right) e^{-j\left[\left(f-f_{0}\right) N / 2+\theta_{1}\right]}+\frac{A_{1}}{2} W\left(f+f_{0}\right) e^{-j\left[\left(f+f_{0}\right) N / 2-\theta_{1}\right]}$

It is hard to make $N \neq m \cdot f_{s} / f_{0}\left(m \in Z^{+}\right)$in sampling; accordingly spectrum leakage becomes inevitably. Mark $\delta$ as frequency deviation and $\Delta f$ as frequency resolution. We can get $f_{0}=(k+\delta) \cdot \Delta f, k$ is a positive integer. Ignoring the negative frequency components, then:

$S_{1 w}(k)=\frac{A_{1}}{2} W\left(f-f_{0}\right) e^{-j\left[\left(f-f_{0}\right) N / 2+\theta_{1}\right]}=\frac{A_{1}}{2} W(-\delta \Delta f) e^{-j\left(\pi \delta+\theta_{1}\right)}$

The phase of $s_{1 w}(n)$ can be estimated as follows: $\varphi_{1}=\theta_{1}+\pi \delta$.

Similarly, the phase of $s_{2 w}(n)$ can be expressed as $\varphi_{2}=\theta_{2}+\pi \delta$.

Then, phase difference can be obtained by the subtraction of the two phases, i.e.,

$\Delta \hat{\theta}=\varphi_{1}-\varphi_{2}=\theta_{1}-\theta_{2}$ 
It seems that the spectrum leakage does not affect the phase difference estimation. But that is not the case; its reasons will be demonstrated in the following.

\section{Influence of Spectrum Leakage}

\subsection{Short-range Leakage: Spectral Lines Offset}

There are two types of spectral leakage: short-range leakage and long-range leakage [12]. Short-range leakage takes place in the main lobe of spectrum, which impact the phase difference estimation by $\delta$. Under the background of the additive noise, sampling sequence can be represented as:

$x(n)=s(n)+z(n), \quad n=0,1, \cdots, N-1$

Where $s(n)$ is signal sampling sequence, and $z(n)$ is white Gaussian noise sequence whose variance is $\sigma_{z}^{2}$. The signal-to-noise ratio expressed as $S N R=A^{2} /\left(2 \sigma^{2}\right)$. Short $x(n)$ by a symmetric window with $N$ sampling point, and then the sampling sequence can be expressed as:

$x_{w}(n)=x(n) w_{N}(n)+z(n) w_{N}(n)$

Ignore the negative frequency components of $x_{w}(k)$, that to say, only consider the first $N / 2$ part of spectrum, there is:

$\vec{X}_{w}(k)=\vec{S}_{N}(k)+\vec{Z}_{N}(k), \quad k=0,1, \cdots N / 2$

Generally, the maximum of amplitude spectrum is obtained on line number $k$ or $k+1$. Mark $k_{r}$ as spectral line number corresponding to the maximum. On the condition of universal $S N R$, it is satisfaction that $Z_{w}^{R}(k) \square S_{w}(k)$ and $Z_{w}^{l}(k) \square S_{w}(k)$ in $k_{r}$. Set $W(f)$ as continuous spectrum of the symmetric window $w(n)$, and then, we can get $S_{w}\left(k_{r}\right)=A \cdot W(\delta) / 2$. According to the formula (7), the phase spectrum equals to:

$X_{w}^{P}\left(k_{r}\right)=\arctan \left(\frac{S_{w}^{I}\left(k_{r}\right)+Z_{w}^{I}\left(k_{r}\right)}{S_{w}^{R}\left(k_{r}\right)+Z_{w}^{R}\left(k_{r}\right)}\right)$

According to the second-order Taylor formula and ignore the higher order dimensionless, formula (8) can be approximately rewrote as:

$X_{w}^{P}\left(k_{r}\right) \approx S_{w}^{P}\left(k_{r}\right)+Z P_{w}\left(k_{r}\right)$

where $Z P_{w}\left(k_{r}\right)=\frac{S_{w}^{R}\left(k_{r}\right)}{S_{w}^{2}\left(k_{r}\right)} Z_{w}^{I}\left(k_{r}\right)-\frac{S_{w}^{I}\left(k_{r}\right)}{S_{w}^{2}\left(k_{r}\right)} Z_{w}^{R}\left(k_{r}\right)=\frac{\cos \left(X_{w}^{P}\left(k_{r}\right)\right) Z_{w}^{I}\left(k_{r}\right)-\sin \left(X_{w}^{P}\left(k_{r}\right)\right) Z_{w}^{R}\left(k_{r}\right)}{S_{w}\left(k_{r}\right)}$

Mark $P w$ as average power of the window. As a result of the real and imaginary part of discrete spectrum distribution of white Gaussian noise sequence are subject to $N\left(0, \sigma^{2} P w / 2 N\right)$ and arbitrary two spectral lines of real part and imaginary part are independence with each other, it's not difficult to know:

$E\left(Z_{w}^{R}(k)\right)=E\left(Z_{w}^{I}\left(k_{r}\right)\right)=0, \operatorname{var}\left(Z_{w}^{R}(k)\right)=\operatorname{var}\left(Z_{w}^{I}\left(k_{r}\right)\right)=\sigma^{2} P w / 2 N$

Thus, taking the $S N R$ definition and symmetric window spectrum into consideration, we can get: 


$$
E\left(X_{w}^{P}\left(k_{r}\right)\right)=S_{w}^{P}\left(k_{r}\right), \operatorname{var}\left(X_{w}^{P}\left(k_{r}\right)\right)=\frac{\sigma^{2} P w}{2 N \cdot S_{w}^{2}\left(k_{r}\right)}=\frac{P w}{N \cdot S N R \cdot W^{2}(\delta)}
$$

Using $S_{1 w}^{R}\left(k_{r}\right)$ and $S_{2 w}^{R}\left(k_{r}\right)$ as denotations of the two signal phase spectrum respectively, we can obtain the variance of phase difference estimation, as follows:

$$
\operatorname{var} \Delta \hat{\theta}=\operatorname{var} S_{1 w}^{P}\left(k_{r}\right)+\operatorname{var} S_{1 w}^{P}\left(k_{r}\right)=\frac{2 P w}{N \cdot S N R \cdot W^{2}(\delta)}
$$

Obviously, the precision of DFT-based phase difference estimation relates to $S N R$, shape and length of the symmetric window and frequency deviation.

\subsection{Long-Range Leakage: Negative Frequency Interference}

Long-range spectrum leakage refers to the side-lobe spectrum leakage, which may cause lines interference. For dense multi-frequency signals, it is necessary to zoom in spectrum before phase difference estimation. For single frequency signal, long range spectrum leakage will cause negative spectrum peak superposition to positive spectrum, namely the negative frequency interference, when the signal frequency is very low or close to the Nyquist frequency. This phenomenon also takes place when sampling point only a little while. Here the method described by formula (2-4) possesses a larger estimation error thanks to the overlooking of negative frequency components. Denote $\theta_{k}$ and $\theta_{k+1}$ as the phases located the maximum and the sub-maximum spectrum line respectively, and then, we commonly deem that there is interference spectrum when:

$$
|| \theta_{k}-\theta_{k+1}|-\pi|>\varepsilon
$$

where $\varepsilon$ is a little positive number.

The phenomenon of negative spectrum interference is shown in Figure 1. The real frequency is included in the range of $(0, \pi)$, named positive frequency range. Range $(-\pi, 0)$ and range $(\pi, 2 \pi)$ are symmetrical spectrum image, called negative frequency range. When the signal frequency is low or close to the Nyquist frequency, spectrum peak $A$ closes to the ends of range $(0, \pi)$ and side-lobe interference increases rapidly, as shown in (b). When position of spectral peak $A$ is very close to $\omega=0$ or $\omega=\pi$, $A$ and $A^{\prime}$ will overlap and result in main lobe interference, as shown in (c).
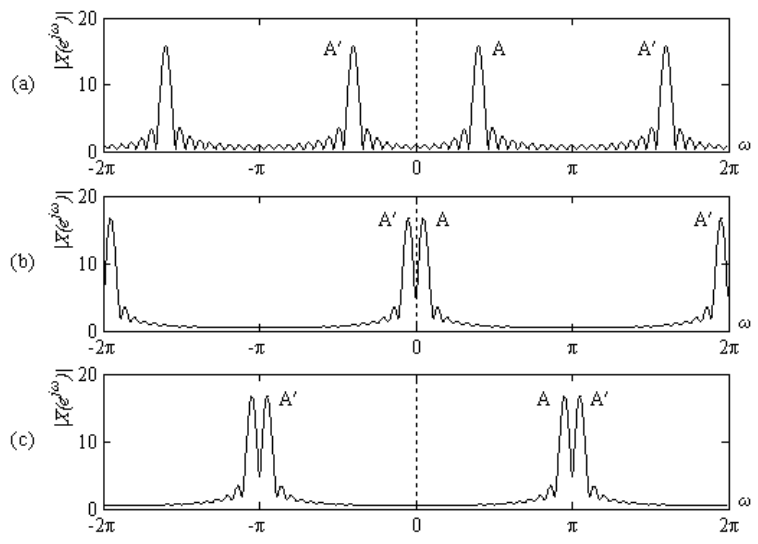

Figure 1. Spectrum with Negative Frequency Interference 


\section{The Proposed Method}

\subsection{Principle}

When a small number of sampled data are taken in the DFT calculation, the negative frequency interference becomes remarkable, that brings about significant errors in phase difference estimation. Therefore, both positive and negative frequency should be taken into consideration when calculate the phase difference. In order to improve the accuracy of phase difference measurement, a method with spectrum leakage considered is proposed. Take the negative frequencies into account, formula (2) equals to:

$$
\begin{aligned}
S_{1}\left(k_{0}\right) & =\sum_{n=0}^{N-1} \frac{A_{1}}{2} e^{j\left[2 \pi\left(k_{0}+\delta\right) \cdot \frac{n}{N}+\theta_{1}\right]} \cdot e^{-j \frac{2 \pi}{N} n k_{0}}+\sum_{n=0}^{N-1} \frac{A_{1}}{2} e^{-j\left[2 \pi\left(k_{0}+\delta\right) \cdot \frac{n}{N}+\theta_{1}\right]} \cdot e^{-j \frac{2 \pi}{N} n k_{0}} \\
& =\frac{A_{1}}{2} e^{j \theta_{1}} \sum_{n=0}^{N-1} e^{j \frac{2 \pi}{N} n \delta}+\frac{A_{1}}{2} e^{-j \theta_{1}} \sum_{n=0}^{N-1} e^{-j \frac{2 \pi}{N} n\left(2 k_{0}+\delta\right)}
\end{aligned}
$$

If rectangular window been used, we can deduce the following equation:

$\tan \left(\theta_{1}+\pi \delta\right)=\frac{c_{2} \cdot \tan \varphi_{1 w}}{c_{1}-c_{3} \cdot \tan \varphi_{1 w}}$

where $\varphi_{1}$ is the phase

of $s_{1}\left(k_{0}\right), c_{1}=\sin \left(2 \pi k_{0} / N\right), c_{2}=\sin \left[2 \pi\left(k_{0}+\delta\right) / N\right], c_{3}=2 \sin (\pi \delta / N) \sin \left[\pi\left(2 k_{0}+\delta\right) / N\right]$.

Similarly, for $s_{2}(n)$

$\tan \left(\theta_{2}+\pi \delta\right)=\frac{c_{2} \cdot \tan \varphi_{2 w}}{c_{1}-c_{3} \cdot \tan \varphi_{2 w}}$

where $\varphi_{2}$ is the DFT phase of $s_{2}(n)$ at $k_{0}$.

On the basis of (15) and (16), the formula for phase difference calculation is deduced as:

$\Delta \theta=\arctan \left[\frac{c_{1} c_{2}\left(\tan \varphi_{2 w}-\tan \varphi_{1 w}\right)}{c_{1}^{2}-c_{1} c_{3}\left(\tan \varphi_{1 w}+\tan \varphi_{2 w}\right)+\left(c_{2}^{2}+c_{3}^{2}\right) \tan \varphi_{1 w} \tan \varphi_{2 w}}\right]$

If the Hanning window is used, we can obtain another expression for phase difference calculation:

$\Delta \theta=\arctan \left[\frac{c_{4}\left(\tan \varphi_{2 w}-\tan \varphi_{1 w}\right)}{1+c_{4}^{2} \tan \varphi_{1 w} \tan \varphi_{2 w}}\right]$

where, $c_{4}=\frac{\sin \left[2 \pi\left(k_{0}+\delta\right) / N\right]}{\sin \left(2 \pi k_{0} / N\right)} \cdot \frac{D_{1}}{D_{2}}$,

$D_{1}=1+\cos (2 \pi / N)-2 \cos (\pi \delta / N) \cdot \cos \left[\pi\left(2 k_{0}+\delta\right) / N\right] \cos \left(2 \pi k_{0} / N\right)$

$D_{2}=1+\cos (2 \pi / N)-2 \cos (\pi \delta / N) \cdot \cos \left[\pi\left(2 k_{0}+\delta\right) / N\right] \cos \left[2 \pi\left(k_{0}+\delta\right) / N\right]$

\subsection{Recursive DFT}

Recursive DFT algorithm introduced in this paper is based on the trigonometric function. Supposing that we have obtained a sampling sequence $x(n)$ at the point of $m$, then $x(n)=[x(0), x(1), \cdots, x(N-1)]$ and its DFT is: 


$$
\begin{gathered}
X(k)=\sum_{n=0}^{N-1} x(n) e^{-j \frac{2 \pi}{N} n k}=\sum_{n=0}^{N-1} x(n)\left(\cos \frac{2 \pi}{N} n k-j \sin \frac{2 \pi}{N} n k\right)=A_{m}(k)-j B_{m}(k) \\
\text { where } A_{m}(k)=\sum_{n=0}^{N-1} x(n) \cos \frac{2 \pi}{N} n k, B_{m}(k)=\sum_{n=0}^{N-1} x(n) \sin \frac{2 \pi}{N} n k .
\end{gathered}
$$

At the point of $m+1$, the sampling sequence $x(n)$ is updated by $x(N)$ with $x(0)$ discarded. Here, the DFT of the updated $x(n)$ are as follows:

$$
X(k)=\sum_{n=1}^{N} x(n) e^{-j \frac{2 \pi}{N}(n-1) k}=\sum_{n=1}^{N} x(n)\left(\cos \frac{2 \pi}{N}(n-1) k-j \sin \frac{2 \pi}{N}(n-1) k\right)=A_{m+1}(k)-j B_{m+1}(k)
$$

where

$$
\begin{aligned}
A_{m+1}(k) & =\sum_{n=1}^{N} x(n) \cos \frac{2 \pi}{N}(n-1) k \\
& =\sum_{n=1}^{N} x(n)\left[\cos \frac{2 \pi}{N} n k \cos \frac{2 \pi}{N} k+\sin \frac{2 \pi}{N} n k \sin \frac{2 \pi}{N} k\right] \\
& =\cos \frac{2 \pi}{N} k\left[x(N) \cos \frac{2 \pi}{N} N k+\sum_{n=1}^{N-1} x(n) \cos \frac{2 \pi}{N} n k\right]+\sin \frac{2 \pi}{N} k\left[x(N) \sin \frac{2 \pi}{N} N k+\sum_{n=1}^{N-1} x(n) \sin \frac{2 \pi}{N} n k\right] \\
& =\cos \frac{2 \pi}{N} k\left[x(N)+A_{m}(k)-x(0)\right]+\sin \frac{2 \pi}{N} k \cdot B_{m}(k)
\end{aligned}
$$

Similarly, $B_{m+1}(k)=\cos \frac{2 \pi}{N} k \cdot B_{m}(k)-\sin \frac{2 \pi}{N} k \cdot\left[x(N)+A_{m}(k)-x(0)\right]$

Apparently, when a new point been taken into the sampling sequence, there is a recursive relationship between DFTs of the two sequences.

\subsection{Process}

According to the principle, process of the advanced method can be summarized as follows:

Step1: Sample two signals synchronously and intercept data with the same window length $N$.

Step2: Calculate the DFTs of sampling signals $s_{1 w}(n)$ and $s_{2 w}(n)$.

Step3: Correct the spectrums by interpolation algorithm [13] and figure out $\delta$.

Step4: Calculate $A_{m}(k)$ and $B_{m}(k)$.

Step5: Calculate the phase difference by Eq. (17) or Eq. (18).

\section{Precision}

DFT of the signal defined by (5) can be express as:

$X_{1}(k)=S_{1}(k)+Z_{1}(k)=A_{k} e^{j \varphi_{k}}+b e^{j \varphi_{2}}, k=0,1, \cdots, N-1$

where $S_{1}(k)$ and $z_{1}(k)$ are the DFT transforms of $s_{\pi_{1}}(n)$ and $z_{1}(n), A_{k}, b$ and $\varphi_{k}, \varphi_{2}$ are the amplitude and phase respectively, $Z_{1}(k)=\sum^{N-1} z_{1}(n) \cdot e^{-j \frac{\pi}{N} n k}$. As $z_{1}(n)$ is a Gaussian white noise, $Z_{1}(k)$ also obey Gaussian distribution, whose variance is $\operatorname{var}\left[Z_{1}(k)\right]=N \sigma_{2}^{2}$. For white noise, there are $E\left[z_{1}(m) z_{1}(n)\right]=0$ when $m \neq n$. Therefore, autocorrelation of $Z_{1}(k)$ can be simplified as: 
$E\left[Z_{1}(k) \cdot Z_{1}^{*}(l)\right]=\sum_{n=0}^{N-1} E\left[z_{1}(n) z_{1}(n)\right] \cdot e^{j \frac{2 \pi}{N}(l-k) n}=\sigma_{z}^{2} \cdot \sum_{n=0}^{N-1} e^{j \frac{2 \pi}{N}(l-k) n}= \begin{cases}N \sigma_{z}^{2}, & \text { when } l=k \\ 0, & \text { when } l \neq k\end{cases}$

Although $z_{1}(k)$ is a linear combination of the same set of random variables, there is a lack of correlation in $Z_{1}(k)$ as a result of the orthogonal feature of DFT basis function. Thereby $Z_{1}(k)$ is complex Gaussian white noise, with $E\left[b \cos \varphi_{2}\right]=E\left[b \sin \varphi_{2}\right]=E\left[Z_{1}(k)\right]=0$ and $\operatorname{var}\left[b \cos \varphi_{z}\right]=\operatorname{var}\left[b \sin \varphi_{z}\right]=\operatorname{var}\left[Z_{1}(k)\right] / 2=N \sigma_{z}^{2} / 2$.

At the maximum $k_{0}$, DFT transform of $x_{1}(n)$ can be represented as:

$R_{1}\left(k_{0}\right)=S_{1}\left(k_{0}\right)+Z_{1}\left(k_{0}\right)=A_{k 1} e^{j \varphi_{f 1}}+b e^{j \varphi_{z}}$

where $A_{k 1}, \varphi_{f 1}$ are the amplitude and phase of $S_{1}\left(k_{0}\right)$ respectively, and

$A_{k 1}^{2}=A_{1}^{2} \sin ^{2} \pi \delta\left[\sin \left(\theta_{1}+\pi \delta\right)+\cos \left(\theta_{1}+\pi \delta\right) c_{f 2} / c_{f 3}\right]^{2}+A_{1}^{2} \sin ^{2} \pi \delta \sin ^{2}\left(\theta_{1}+\pi \delta\right) c_{f 1}^{2} / c_{f 3}^{2}$

Then, we rewrite (23) as:

$$
\begin{aligned}
R_{1}\left(k_{0}\right) & =A_{k 1} e^{j \varphi_{f_{1}}}\left[1+\frac{b}{A_{k 1}} e^{j\left(\varphi_{z}-\varphi_{f 1}\right)}\right] \\
& =A_{k 1} e^{j \varphi_{f 1}}\left[1+\frac{b}{A_{k 1}} \cos \left(\varphi_{z}-\varphi_{f_{1}}\right)+j \frac{b}{A_{k 1}} \sin \left(\varphi_{z}-\varphi_{f_{1}}\right)\right] \\
& =A_{k 1} \sqrt{1+\left(\frac{b}{A_{k 1}}\right)^{2}+\frac{2 b}{A_{k 1}} \cos \left(\varphi_{z}-\varphi_{f_{1}}\right)} \cdot e^{j \varphi_{f_{1}}^{\prime}}
\end{aligned}
$$

where $\varphi_{f 1}^{\prime}$ is the phase of $S_{1}\left(k_{0}\right)$, and

$$
\varphi_{1}^{\prime}=\varphi_{1}+\arctan \left[\frac{b \sin \left(\varphi_{z}-\varphi_{1}\right) / A_{k 1}}{1+b \cos \left(\varphi_{z}-\varphi_{1}\right) / A_{k 1}}\right]
$$

It is generally believed that $b / A_{k 1}<<1$ when $S N R$ is not particularly low and $N$ is sufficient large. Therefore:

$$
\varphi_{f 1}^{\prime} \approx \varphi_{f 1}+\arctan \left[\frac{b}{A_{k 1}} \sin \left(\varphi_{z}-\varphi_{f 1}\right)\right] \approx \varphi_{f 1}+\varphi_{z 1}
$$

where $b$ and $\varphi_{z}$ are stochastic while $A_{k 1}$ and $\varphi_{f 1}$ are invariable. $\varphi_{z 1}=b \sin \left(\varphi_{z}-\varphi_{f 1}\right) / A_{k 1}$ is Gaussian white noise, and $\operatorname{var}\left[\varphi_{z 1}\right]=N \sigma_{z}^{2} / 2 A_{k 1}^{2}$.

Similarly, we can obtain:

$$
\varphi_{f 2}^{\prime}=\varphi_{f 2}+\varphi_{z 2}
$$

where $\varphi_{z 2}=b \sin \left(\varphi_{z}-\varphi_{f 2}\right) / A_{k 2}$ is the phase of $S_{2}\left(k_{0}\right)$ and obey Gaussian distribution, with $\operatorname{var}\left[\varphi_{z 2}\right]=N \sigma_{z}^{2} / 2 A_{k 2}^{2}$. Then the phase difference can be get by:

$\Delta \theta=\arctan \left[\frac{\beta_{f 3}^{\prime}}{\beta_{f 1}^{\prime}+\beta_{f 2}^{\prime}}\right]$

where

$\beta_{f 1}^{\prime}=c_{f 1}^{2}-c_{f 1} c_{f 3}\left(\tan \varphi_{f 1}^{\prime}+\tan \varphi_{f 2}^{\prime}\right), \beta_{f 2}^{\prime}=\left(c_{f 2}^{2}+c_{f 3}^{2}\right) \tan \varphi_{f 1}^{\prime} \tan \varphi_{f 2}^{\prime}, \beta_{f 3}^{\prime}=c_{f 1} c_{f 2}\left(\tan \varphi_{f 2}^{\prime}-\tan \varphi_{f 1}^{\prime}\right)$.

Set $\mathbf{T}=\left[\begin{array}{ll}T_{1} & T_{2}\end{array}\right]^{T}=\left[\begin{array}{ll}\varphi_{f 1}^{\prime} & \varphi_{f 2}^{\prime}\end{array}\right]^{T}$ and $E[\mathbf{T}]=\left[\begin{array}{ll}\varphi_{f 1} & \varphi_{f 2}\end{array}\right]^{T}$, then $\Delta \theta=f(\mathbf{T})$. At the point of $\mathbf{T}_{0}=E[\mathbf{T}]=\left[\begin{array}{ll}\varphi_{f 1} & \varphi_{f 2}\end{array}\right]^{T}=\left[\begin{array}{ll}\mu_{1} & \mu_{2}\end{array}\right]^{T}=\boldsymbol{\mu}$, the first order Taylor series expansion of $f(\mathbf{T})$ is: 
$\Delta \theta=f(T) \approx f(\mu)+\sum_{k=1}^{2} \frac{\partial f}{\partial T_{k}} \mid \mathbf{T}=\mathbf{T}_{0}\left(T_{k}-\mu_{k}\right)$

Then

$$
\begin{aligned}
& E[\Delta \theta]=f(\boldsymbol{\mu})=\arctan \left[\frac{c_{f 1} c_{f 2}\left(\tan \varphi_{f 2}-\tan \varphi_{f 1}\right)}{\beta_{f 1}+\beta_{f 2}}\right] \\
& \operatorname{var}[\Delta \theta]=E\left\{\left[f(\mu)+\left.\frac{\partial f}{\partial \mathbf{T}}\right|_{\mathbf{T}=\boldsymbol{\mu}} ^{T}(\mathbf{T}-\boldsymbol{\mu})-E[\Delta \theta]\right]^{2}\right\}=\left.\frac{\partial f}{\partial \mathbf{T}}\right|_{\mathbf{T}=\boldsymbol{\mu}} ^{T} \mathbf{C}_{T} \frac{\partial f}{\partial \mathbf{T}} \mid \mathbf{T}=\boldsymbol{\mu}
\end{aligned}
$$

where $\mathbf{C}_{T}$ is the covariance matrix of matrix $\mathbf{T}$.

$\left.\frac{\partial f}{\partial T_{1}}\right|_{T_{1}=\mu_{1}}=\Delta_{f 1}=\frac{-c_{f 1} c_{f 2} \sec ^{2} \varphi_{f 1}\left[c_{f 2}^{2} \tan ^{2} \varphi_{f 2}+\left(c_{f 3} \tan \varphi_{f 2}-c_{f 1}\right)^{2}\right]}{\beta_{f 3}^{2}+\left[\beta_{f 1}+\beta_{f 2}\right]^{2}}$

$\left.\frac{\partial f}{\partial T_{2}}\right|_{T_{2}=\mu_{2}}=\Delta_{f 2}=\frac{c_{f 1} c_{f 2} \sec ^{2} \varphi_{f 2}\left[c_{f 2}^{2} \tan ^{2} \varphi_{f 1}+\left(c_{f 3} \tan \varphi_{f 1}-c_{f 1}\right)^{2}\right]}{\beta_{f 3}^{2}+\left[\beta_{f 1}+\beta_{f 2}\right]^{2}}$

$\left.\frac{\partial f}{\partial \mathbf{T}}\right|_{\mathbf{T}=\boldsymbol{\mu}}=\left[\begin{array}{ll}\Delta_{f 1} & \Delta_{f 2}\end{array}\right]^{T}$

Typically, Gauss white noise is unrelated with each other. Thereby $\varphi_{z 1}$ and $\varphi_{z 2}$ is not relevant too. So

$\mathbf{C}_{T}=\left[\begin{array}{cc}N \sigma_{z}^{2} / 2 A_{k 1}^{2} & 0 \\ 0 & N \sigma_{z}^{2} / 2 A_{k 2}^{2}\end{array}\right]$

Then, we can deduce that

$\operatorname{var}[\Delta \theta]=\frac{N \sigma_{z}^{2}}{2}\left(\frac{\Delta_{f 1}^{2}}{A_{k 1}^{2}}+\frac{\Delta_{f 2}^{2}}{A_{k 2}^{2}}\right)$

\section{Experiment Results}

To verify the influence of spectral leakage and evaluate the performance of the proposed method, computer simulations are carried out, assuming that the signals are single-frequency real signals with white Gaussian noise. In simulations, the initialization of phase difference is $\Delta \theta=4.1^{\circ}$.

\subsection{Influence of Frequency Deviation $|\delta|$ and Windows}

In simulations, the signal frequency equals $198 \mathrm{~Hz}$, the sampling frequency equals $2000 \mathrm{~Hz}, S N R=20 \mathrm{~dB} . N=1024$. Rectangular window, Hanning window and Hamming window, are used respectively in simulations. Phase differences are calculated 200 times independently by the DFT-based method with ratio correction method as comparison. The root mean square error (RMSE) of phase difference estimation and the relationship between the results and deviation $\delta$ are shown in Figure 2.

The theoretic values of RMSE are denoted by lines while simulation results are shown by discrete points. As can be seen from the Figure 2, the simulation results agreed with the theoretical value. RMSE of the uncorrected DFT-based method deduce with the deviation $\mid \delta$ | closing to zero. We can also come to the conclusion that RMSE with the Hanning window and Hamming window are similar. When $|\delta|$ is small, the RMSE with a rectangular window is smaller compared with Hanning window or Hamming window. However, the conclusion reverses when $|\delta|$ is remarkable. After corrected, the RMSE is 
always in a near constant value and has nothing to do with $|\delta|$. The constant value (about $0.253^{\circ}$ ) is determined by $S N R$ and $N$.
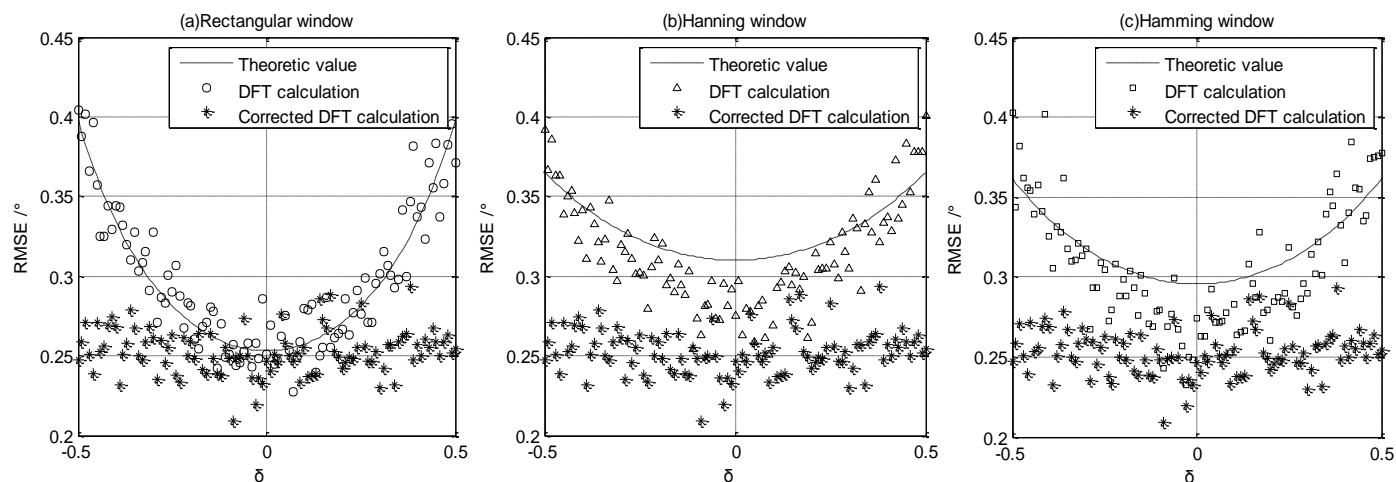

Figure 2. Relationship between RMSE and the Deviation

\subsection{Influence of the SNR and Sampling Length}

Under the condition of $N=1024, k_{0}=200$ and $\delta=0.4$, the relationship between the RMSE and SNR is show in Figure 3. The phenomenon of RMSE changes with sampling length under the condition of $k_{0}=30, \delta=0.4$ and $S N R=20 \mathrm{~dB}$ is also denoted in Figure 3. It can be seen that the simulation results and formula calculation results are consistent. What's more, the higher $S N R$, the smaller the RMSE of phase difference estimation.

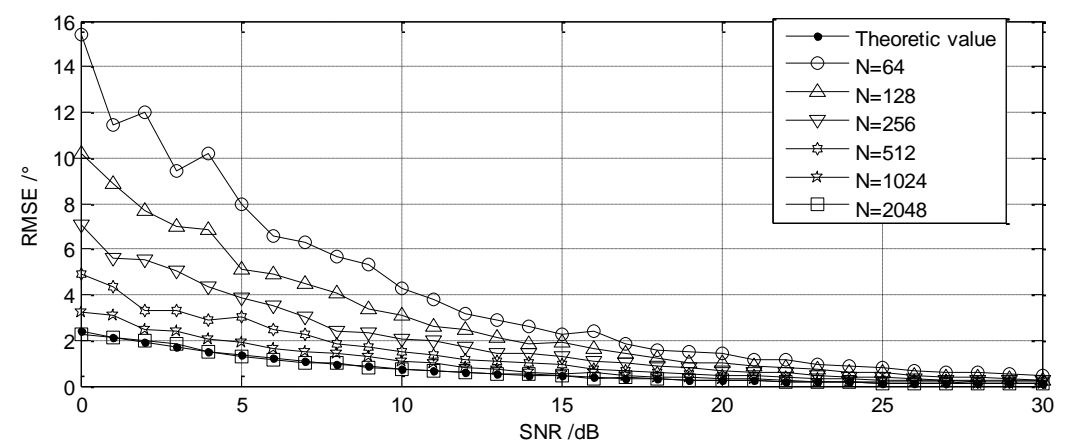

Figure 3. Relationship of RMSE with SNR and Sampling Length

\subsection{Precision of the Proposed Method}

In simulations, the number of sampled points equals 1024, the sampling frequency equals $2000 \mathrm{~Hz}$ and the frequency resolution is $\Delta f=f_{s} / N=0.9766 \mathrm{~Hz}$. The windows of rectangular and Hanning are used respectively. In order to visually reflect the inherent law of signal spectrum, signal frequency is selected with frequency resolution as a basic unit. Signal frequency range from $0.5 \Delta f$ to $2.5 \Delta f$ and $497.5 \Delta f$ to $499.5 \Delta f$, step length equals $0.05 \Delta f$. The phase differences are calculated by the proposed method and the relative errors of calculation are shown in Figure 4. Apparently, the proposed method has higher precision than universal DFT-based method. The error of DFT method fell sharply close to the lower limit of double precision arithmetic when the relative frequency $f_{0} / f_{d}$ is an integer. This is because side lobe of the negative frequency in positive spectrum is exactly zero and doesn't have any impact on positive spectrum. 


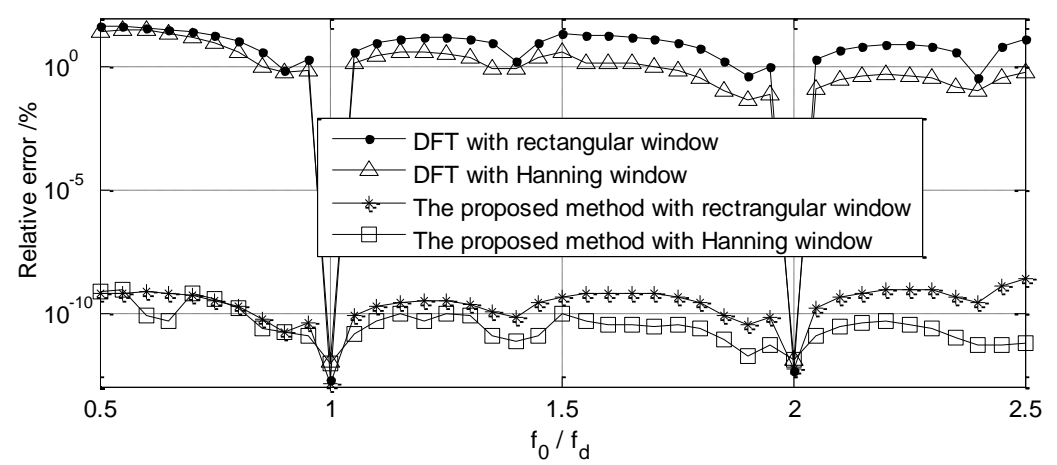

Figure 4. Relative Error of Phase Difference Measurement

\subsection{Application in CMF}

To experimentally validate the proposed method, a system for CMF signal processing has been developed, as shown in Figure 5. We select a kind of CMF(F200S) with a 1700R transmitter. A 4-channal dynamic signal acquisition NI 9234 which operates with sampling rate of $20000 \mathrm{~Hz}$ is used to sample the CMF's oscillation signals. The signal frequency of CMF sensors equals about $198 \mathrm{~Hz}$. Mass flow measured by the scale is deemed as the actual value.

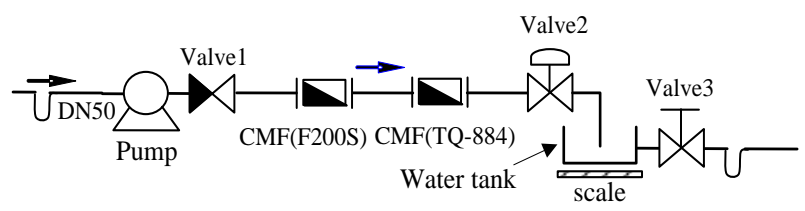

Figure 5. Block of the Experimental System

According to CMF's principle, the mass flow rate is calculated by the time interval $\Delta t$, which depends on the frequency and the phase difference. The SGA is taken as comparisons.

\section{Table 1. Experimental Results}

\begin{tabular}{cccc}
\hline $\begin{array}{c}\text { Mass flow } \\
(\mathrm{kg} / \mathrm{min})\end{array}$ & $\begin{array}{c}\text { Theoretic value of time } \\
\text { interval }(\mathrm{kg} / \mathrm{min})\end{array}$ & $\begin{array}{c}\text { The SGA } \\
(\mathrm{kg} / \mathrm{min})\end{array}$ & $\begin{array}{c}\text { The proposed method } \\
(\mathrm{kg} / \mathrm{min})\end{array}$ \\
\hline 2.28 & 16.4143 & 15.6657 & 16.4623 \\
8.50 & 61.0783 & 58.2380 & 61.2571 \\
16.84 & 120.9653 & 115.2087 & 121.3193 \\
\hline
\end{tabular}

As shown in Table 1, the results of the proposed method are more close to the theoretic values compared with the SGA. Then, we come to the conclusion that the proposed method is effective and practical.

\section{Conclusion}

For phase difference measurement method based on DFT, there is a problem unable to slide over that spectrum leakage has a disadvantage impact on precision. In this paper, we demonstrate how spectrum leakage influence the performance of the DFT based method and put forward a novel method based on recursive DFT with short range leakage and long range leakage both been considered. Simulation and experimental results show that the accuracy of phase difference measurement has largely improved compared with the SGA. Precision of the proposed method can be improved father by selected appropriate 
windows. What's more, the proposed method can also be used to track dynamic phase difference. Further research is under discussion.

\section{Acknowledgment}

The authors greatly appreciate the financial supports from the National Natural Science Foundation of China $(61271449,61302175)$ and the Chongqing Natural Science Foundation of CSCT (CSTC2012jjA40006, CSTC2013jcyjA40030).

\section{References}

[1] A. Martin, D. Wolfgang and R. Alfred, "Coriolis mass flowmeters: Overview of the current state of the art and latest research", Flow Measurement and Instrumentation, vol. 17, (2006), pp. 317-323.

[2] J. J. Zheng and S. B. Yu, "Research of real-time detection technology of phase", Electrical Measurement \& Instrumentation, vol. 40, no. 456, (2003), pp. 29-32.

[3] Y. Q. Tu, T. A. Shen, M. Li and H. T. Zhang, "Research on phase difference measurement algorithm for non-integer period sampling signal based on multi-layer correlation", Chinese Journal of Scientific Instrument, vol. 35, no. 7, (2014), pp. 1578-1585.

[4] Y. Q. Jiang and Y. G. He, "New algorithm for high-accuracy phase difference measurement based on windowed DFT", Journal of Circuits and Systems, vol. 10, no. 2, (2005), pp. 112-116.

[5] J. Li and Y. F. Wang, "DFT phase estimation algorithm and noise sensitive frequency region Journal of Electronics \& Information Technology", vol. 3 no. 9, (2009), pp. 2099-2103.

[6] S. Schuster, S. Scheiblhofer and S. Andreas, "The influence of windowing on bias and variance of DFT-based frequency and phase estimation [J]", IEEE Transactions on Instrumentation and Measurement, vol. 58, no.6, (2009), pp. 1975-1990.

[7] S.S. Young, K.J. Ho, B.W. Weui, Y. Suk and J.A. Se, "A study on the leakage error in the spectrum of acoustic intensity", JSME International Journal Series A, vol. 47, no. 1, (2004), pp.42-46.

[8] N. M. Vu cijak and L. V. Saranovac, "A simple algorithm for the estimation of phase difference between two sinusoidal voltages", IEEE Transactions on Instrumentation and Measurement, vol. 59, no. 12, (2010), pp. 3152-3158.

[9] Y. Q. Tu, H.Y. Yang, H.T. Zhang and X.Y. Liu, "CMF signal processing method based on feedback corrected ANF and Hilbert transformation Measurement Science Review", vol. 14, no. 1, (2014), pp. 41-47.

[10] K. J. $\mathrm{Xu}$ and W. F Xu, "A signal processing method based on AFF and SGA for coriolis mass flowmeters ACTA Metrologica SINICA", vol. 28, no. 1, (2007), pp. 48-51.

[11] Y.Q. Tu, H.T. Zhang, Y.W. Mao, T.A. Shen and H.Y. Yang, "Unbiased phase delay estimator with negative frequency contribution for real sinusoids", Journal of Applied Sciences, vol. 13, no. 8, (2013), pp. 1160-1168.

[12] K. F. Chen, J. T. Jiang and S. Crowsen, "Against the long-range spectral leakage of the cosine window family Computer Physics Communications", vol. 180, (2009), pp. 904-911.

[13] K. F. Chen, J. L. Yang and S. W. Zhang, "Spectrum correction based on the complex ratio of discrete spectrum around the main-lobe", Journal of Vibration Engineering, vol. 21, no. 3,(2008), pp. 314-317.

\section{Authors}

Huiyue Yang, he received his B.S. degree in Automatic from Logistical Engineering University, China, in 2009 and his M.S. degree in Detection Technology and Automation Devices in 2012. Now, he is a Ph.D. candidate in Logistical Engineering University. His main research interests include signal processing, intelligent detection and instrumentation.

Yaqing Tu, he received his B.S. degree in 1984 from automatic control engineering in Chengdu university of Science and Technology, China, M.S. degree in 1991 from automatic control theory and application in Chongqing University, China, and Ph.D. degree in 1994 from precise instrument and machinery in Chongqing University. Now he is a professor and Ph.D. supervisor in Logistical Engineering University. His main research interests include intelligent detection and instrumentation, intelligent automation system. 
International Journal of Signal Processing, Image Processing and Pattern Recognition Vol.8, No.11 (2015) 